\title{
Phosphorylated acidic serine-aspartate-rich MEPE-associated motif peptide from matrix extracellular phosphoglycoprotein inhibits phosphate regulating gene with homologies to endopeptidases on the $X$-chromosome enzyme activity
}

\author{
Shiguang Liu*, Peter S N Rowe*, Luke Vierthaler, Jianping Zhou and L Darryl Quarles \\ The Kidney Institute, University of Kansas Medical Center, 3901 Rainbow Boulevard, MS 3018, Kansas City, Kansas 66160, USA \\ (Requests for offprints should be addressed to S Liu; Email: sliu@kumc.edu) \\ *(S Liu and P S N Rowe contributed equally to this work)
}

\begin{abstract}
Inactivating PHEX (phosphate regulating gene with homologies to endopeptidases on the $\mathrm{X}$ chromosome) mutations cause $\mathrm{X}$-linked hypophosphatemia in humans and mice (Hyp) through overproduction of fibroblast growth factor 23 (FGF23) a phosphaturic factor, by osteocytes. Matrix extracellular phosphoglycoprotein (MEPE) is also elevated in Hyp and other hypophosphatemic disorders. In addition, the administration of an ASARM (acidic serineaspartate rich MEPE-associated motif) peptide derived from MEPE causes phosphaturia and inhibits bone mineralization in mice, suggesting that MEPE also plays a role in phosphate homeostasis. Since recent studies found that MEPE binds specifically to PHEX in vitro, we tested the effect of recombinant-MEPE and its ASARM peptide on PHEX enzyme activity in vitro and FGF23 expression in bone marrow stromal cell cultures ex vivo. We found that both recombinant MEPE and synthetic phosphorylated ASARM
\end{abstract}

peptide (ASARM-PO ${ }_{4}$ ) inhibit PHEX enzyme activities in an in vitro fluorescent-quenched PHEX enzyme activity assay. The ASARM- $\mathrm{PO}_{4}$ peptide inhibits PHEX enzyme activity in a dose-dependent manner with a $K_{\mathrm{i}}$ of $128 \mathrm{nM}$ and $V_{\max -\mathrm{i}}$ of 100\%. Recombinant MEPE also inhibits PHEX activity $\left(K_{\mathrm{i}}=2 \mathrm{nM}\right.$ and $\left.V_{\max -\mathrm{i}}=26 \%\right)$. Long-term bone marrow stromal cell cultures supplemented with $10 \mu \mathrm{M}$ ASARM$\mathrm{PO}_{4}$ peptide resulted in significant elevation of FGF23 transcripts and inhibition of mineralization. These findings suggest that MEPE inhibits mineralization and PHEX activity and leads to increased FGF23 production. The resulting coordination of mineralization and release of a phosphaturic factor by MEPE may serve a physiological role in regulating systemic phosphate homeostasis to meet the needs for bone mineralization.

Journal of Endocrinology (2007) 192, 261-267

\section{Introduction}

Fibroblast growth factor 23 (FGF23) is a novel phosphaturic hormone (Econs \& Drezner 1994, ADHR Consortium 2000, Shimada et al. 2001) predominately produced by osteocytes in bone (Liu et al. 2006b). FGF23 regulates serum phosphate levels by inhibition of renal phosphate reabsorption and by suppression of $1,25(\mathrm{OH})_{2} \mathrm{D}_{3}$ production by the kidney. Overexpression of FGF23 causes hypophosphatemia and reduced $1,25(\mathrm{OH})_{2} \mathrm{D}_{3}$ levels and rickets/osteomalacia (Bai et al. 2004, Larsson et al. 2004, Shimada et al. 2004b). In contrast, FGF23 deficiency (Bai et al. 2003, Shimada et al. 2004a, Liu et al. 2006b) or mutations increasing FGF23 degradation (Larsson et al. 2005) results in hyperphosphatemia, increased serum $1,25(\mathrm{OH})_{2} \mathrm{D}_{3}$ levels, and soft tissue calcifications. FGF23 is a pathological phosphaturic factor in several hereditary hypophosphatemic disorders, including X-linked hypophosphatemia (XLH) and autosomal dominant hypophosphatemic rickets (ADHR). XLH is caused by inactivating mutations of PHEX (phosphate regulating gene with homologies to endopeptidases on the X chromosome; The HYP Consortium 1995), a member of the M13 family of the type II cell-surface zinc-dependent proteases (Rowe et al. 1997). Serum levels of FGF23 are elevated in XLH (Yamazaki et al. 2002, Weber et al. 2003) and Hyp mice (Liu et al. 2003), the murine homologue of XLH. FGF23 is not the substrate for PHEX (Liu et al. 2003, Benet-Pages et al. 2004); instead loss of PHEX function results in overproduction of FGF23 by osteocytes in bone through mechanisms that remain to be defined (Liu et al. 2003, 2006c). ADHR is caused by mutations that prevent the degradation of FGF23 (White et al. 2001).

Matrix extracellular phosphoglycoprotein (MEPE) is a member of the SIBLING protein family (Fisher \& Fedarko 
2003) originally cloned from a patient with tumor-induced osteomalacia (TIO) (Rowe et al. 2000). MEPE is exclusively expressed in osteoblasts, osteocytes, and odontoblasts and markedly up-regulated in Hyp osteoblasts and TIO tumors (Petersen et al. 2000, Rowe et al. 2000, 2004, Argiro et al. 2001, Guo et al. 2002, Fisher \& Fedarko 2003, Gowen et al. 2003). MEPE was originally proposed as candidate for the phosphaturic factor in TIO; however, MEPE deficiency results in accelerated osteoblast-mediated bone formation and mineralization without changes in serum phosphate levels (Gowen et al. 2003, Liu et al. 2005b). These findings suggest that its primary function is to regulate the mineralization process, although administration of recombinant MEPE is reported to induce phosphaturia in mice (Rowe et al. 2004). In addition, MEPE can be cleaved by cathepsin B to release a carboxy-terminal MEPE peptide (ASARM peptide) (Rowe et al. 2004) and the phosphorylated ASARM peptide inhibits mineralization in vivo and in vitro and binds to PHEX (Rowe et al. 2004, 2005), suggesting that MEPE also undergoes proteolytic processing.

MEPE is elevated in the bone of Hyp mice and positively correlated with FGF23 expression (Weber et al. 2003, Liu et al. 2005b), but the relationship between PHEX and MEPE is not clear. Similar to its proposed role in TIO, MEPE was also thought to be a candidate for the phosphaturic factor in XLH/Hyp. However, we have recently demonstrated that the Hyp phosphaturic phenotype is not corrected by transfer of MEPE-deficient mice on to the Hyp mouse background (Liu et al. 2005b), indicating that MEPE is not the phosphaturic factor in this disorder. While these studies indicate that MEPE is not the proximate cause of hypophosphatemia in Hyp mice, it does not preclude an upstream effect of MEPE that is potentially mediated through MEPE binding to PHEX via the MEPE ASARM-motif or a role of MEPE in the mineralization defect in Hyp mice (Rowe et al. 2005).

To further understand the relationship between PHEX, MEPE, and FGF23 in causing hypophosphatemia, we tested the ability of recombinant-MEPE and ASARM peptides to inhibit PHEX enzyme activity in vitro. We also determined whether MEPE and ASARM-PO 4 peptides were able to up-regulate FGF23 expression and inhibit mineralization in a bone marrow stromal cell culture system.

\section{Materials and Methods}

\section{Assay of SecPHEX endopeptidase activity}

A quenched fluorescence enzymatic assay used to measure PHEX endopeptidase activity was kindly offered by Dr Philippe Crine from Enobia Inc., Montreal, Canada (Campos et al. 2003). In this assay, a secreted form PHEX (SecPHEX) and a synthetic substrate (Abz)-GFSDYK-(Dnp) were used. The synthetic peptide substrate was labeled with a fluorogenic reagent aminobenzoic acid (Abz) in the amino terminus and a quenching moiety dinitrophenyl (Dnp) in the carbonic terminus. The secPHEX dose-dependent activity was tested from concentrations of 10 to $100 \mathrm{ng}$ with $10 \mu \mathrm{M}$ substrate. The reaction was followed by measuring the fluorescence from excitation at $320 \mathrm{~nm}$ and emission at $420 \mathrm{~nm}$ in a Synergy HT Multi-Detection Microplate Reader (BioTek Instruments, Inc., Winooski, VT, USA). Full-length insect expressed human MEPE was purified and expressed as described previously (Rowe et al. 2004, 2005). Synthetic peptides derived from MEPE sequence, including MEPEarginine glycine aspartate motif (RGD) peptide, phosphorylated ASARM (ASARM-PO ${ }_{4}$ ), and non-phosphorylated ASARM peptide (Multiple Peptide Systems, Inc., San Diego, CA, USA) were used in the inhibition assay (Rowe et al. 2005). PHEX activities were expressed as an initial speed rate calculated by fitting the steepest linear slope over at least eight adjacent reading values. The slope was converted into nanomoles of substrate hydrolyzed per minute and kinetic parameters, $K_{\mathrm{i}}$ and $V_{\max -\mathrm{i}}$, were calculated by the non-linear regression data analysis GraphPad Prism 4 software (GraphPad Software, Inc., San Diego, CA, USA).

\section{Animal care and genotyping}

The FGF23-deficient/reporter mouse model was created by knocking in an enhanced green fluorescent protein (eGFP) construct following the adenine thymine guanine (ATG) in exon 1 of the FGF23 gene as previously reported (Liu et al. 2006b) and was crossed and maintained on C57BL/6J background. This eGFP reporter mouse was also transferred onto Hyp background. The heterozygous FGF23-deficient/ reporter mouse will express eGFP under control of endogenous FGF23 promoter and mimic the expression pattern of FGF23. Genomic DNA tissue was extracted and purified from the tail of each mouse using a QIAGEN DNeasy Tissue Kit (QIAGEN Inc). Genotypes were determined by PCR as previously reported (Liu et al. 2006b).

All mice were fed with a standard rodent diet (8604 Harlan Teklad Rodent Diet) containing $1 \cdot 01 \%$ phosphorus and $1 \cdot 36 \%$ calcium (Harlan Teklad, Madison, WI, USA) and tap water. All mice were maintained and used in accordance with recommendations in the Guide for the Care and Use of Laboratory Animals, prepared by the Institute on Laboratory Animal Resources, National Research Council (DHHS Publ. NIH 86-23, 1985).

\section{Bone marrow stromal cell (BMSC) culture}

BMSCs from long bones isolated from 6- to 8- week-old male FGF23-deficient/eGFP reporter mice on both wildtype (WT) and Hyp background were cultured as previously described (Liu et al. 2006b). Briefly, bone marrow cells were isolated and plated in $35 \mathrm{~mm}$ dishes at a density of $7 \times 10^{6}$ per $35 \mathrm{~mm}$ plate and grown in 2-minimum essential medium ( $\alpha$-MEM; Invitrogen) containing 10\% fetal bovine serum (FBS) for 5 days and then switched to the differentiation medium ( $\alpha$-MEM containing 10\% FBS supplemented with 
$5 \mathrm{mmol} / 1, \beta$-glycerophosphate, and $25 \mu \mathrm{g} / \mathrm{ml}$ ascorbic acid) with and without phosphorylated ASARM peptide at $10 \mu \mathrm{M}$ concentration. At day 14, pictures of the cells were taken under bright field and fluorescent light microscopy. Cells were then either stained with Alizarin red-S or used for RNA extraction. Alizarin red-S stain was extracted with 10\% cetylpyridinium chloride and quantified by absorbance measurement at $562 \mathrm{~nm}$ as previously described (Liu et al. 2005a).

\section{$R N A$ isolation and quantitative RT-PCR}

Total RNAs were extracted from cultured cells with Trizol (Invitrogen) and then treated with RNase-free DNase using an RNeasy column (QIAGEN Inc.). First strand cDNA was synthesized using iScript cDNA Synthesis Kit (Bio-Rad). Total RNA $(1 \mu \mathrm{g})$ was used in each $20 \mu$ reverse transcription reaction. Input RNAs (400 and $20 \mathrm{ng}$ ) were used to amplify FGF23 and cyclophilin A respectively. The primers used for real-time quantitative RT-PCR are 5' ${ }^{\prime}$ TGTCGCAGAAGCATCA $-3^{\prime}$ and $5^{\prime}$-GTGGGCGAACAGTGTAGAA- $3^{\prime}$ for FGF23 and $5^{\prime}$-CTGCACTGCCAAGACTGAAT- $3^{\prime}$ and 5'-CCACAATGTTCATGCCTTCT-3' for cyclophinin A. The iCycler iQ Real-Time PCR Detection System and iQ SYBR Green Supermix (Bio-Rad) were used for real-time PCR analysis as previously reported (Liu et al. 2006b).

\section{Results}

Recombinant MEPE and ASARM-PO 4 inhibit PHEX enzyme activities

To evaluate the functional significance of our previously reported MEPE and MEPE ASARM-peptide (phosphorylated and non-phosphorylated) interactions with PHEX (Rowe et al. 2005), we examined if addition of recombinant MEPE or its ASARM peptide (phosphorylated and nonphosphorylated) inhibited PHEX enzymatic activity in vitro. For these studies, we used the previously reported soluble secreted recombinant PHEX enzyme and a synthetic fluorescent-quenched peptide that is specifically cleaved by PHEX (Campos et al. 2003). We found that both recombinant MEPE (Fig. 1) and synthetic phosphorylated ASARM peptide (ASARM-PO 4 ; Fig. 2) inhibit PHEX enzyme activities. Recombinant full-length MEPE significantly inhibited PHEX activity at concentrations of $2 \mathrm{nM}$ in a dose-dependent and saturable manner. The $K_{\mathrm{i}}$ and $V_{\max -\mathrm{i}}$ were $2 \mathrm{nM}$ and $26 \%$ respectively (Fig. 1). Synthetic ASARM$\mathrm{PO}_{4}$ peptide (derived from the C-terminal MEPE ASARMmotif) also resulted in a potent inhibition of PHEX activity in vitro with a $K_{\mathrm{i}}$ and $V_{\max -\mathrm{i}}$ of $129 \mathrm{nM}$ and $100 \%$ respectively (Fig. 2). Specifically, dose-, time-dependent, and saturable inhibition of PHEX activity was observed at $\mathrm{nM}$ concentrations for both recombinant MEPE and ASARM-PO Control RGD peptide (Fig. 2) and non-phosphorylated
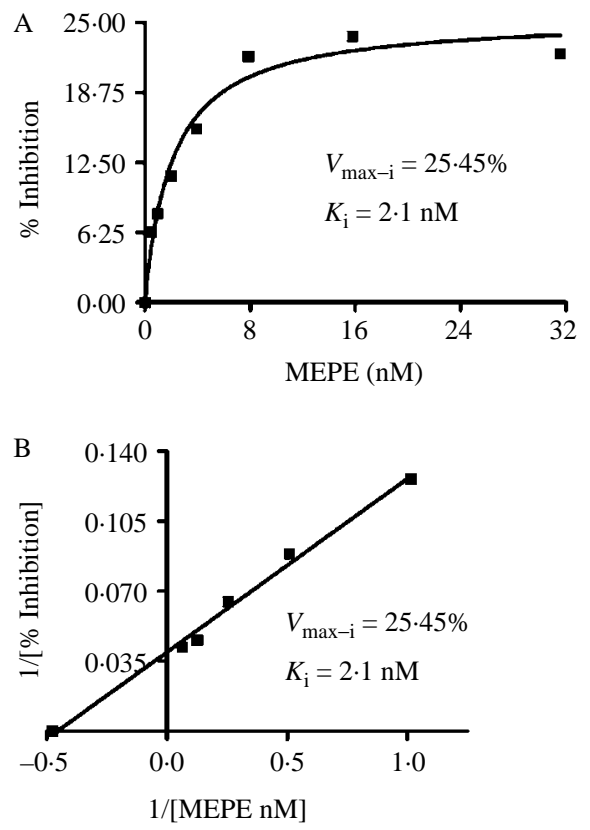

Figure 1 Kinetics of inhibition of PHEX enzymatic activity by recombinant MEPE. Michaelis-Menton graph (A) and corresponding Lineweaver-Burke plot (B) showing percentage inhibition of secPHEX activity as measured using the quenched fluorescence enzymatic assay (see Materials and Methods). Percentage inhibition represents the percentage change in enzyme velocity per unit time relative to control recombinant secPHEX in the absence of inhibitor peptides ( $0 \%$ inhibition of activity). Recombinant insect expressed MEPE-inhibited secPHEX activity in a dose-dependent and saturable manner with a maximum inhibition $\left(V_{\max -i}\right)$ of $25.45 \%$ and a $K_{\mathrm{i}}$ of $2 \cdot 1 \mathrm{nM}$. Each experimental dose point is statistically significant by one-way ANOVA analysis at $P$ value $<0 \cdot 05$.

ASARM peptide (data not shown) had no effect on PHEX activity at micromolar levels. The inhibitory effects of MEPE and ASARM-PO ${ }_{4}$ on PHEX enzyme activity are consistent with previously reported surface plasmon resonance binding studies (Rowe et al. 2006).

\section{ASARM-PO ${ }_{4}$ stimulates FGF23 transcript and inhibits mineralization in BMSC cultures}

Next, we explored whether ASARM-PO ${ }_{4}$ inhibition of PHEX activity has biological significance. To evaluate the effects of ASARM-PO ${ }_{4}$ on FGF23 production by osteoblasts, we incubated $10 \mu \mathrm{M}$ ASARM-PO ${ }_{4}$ with BMSCs derived from FGF23-deficient/eGFP reporter mice, which express eGFP under the control of the endogenous FGF23 promoter. For controls, we assessed BMSCs not treated with peptide (normal controls) and BMSCs from Hyp mice (positive controls), which are PHEX deficiency and exhibit elevated FGF23 expression (Liu et al. 2006b). Incubation of these cells for 14 days in the presence of $10 \mu \mathrm{M}$ ASARM-PO 4 peptide resulted in a significant twofold increase in FGF23 mRNA levels compared with untreated control cultures (Fig. 3A). 

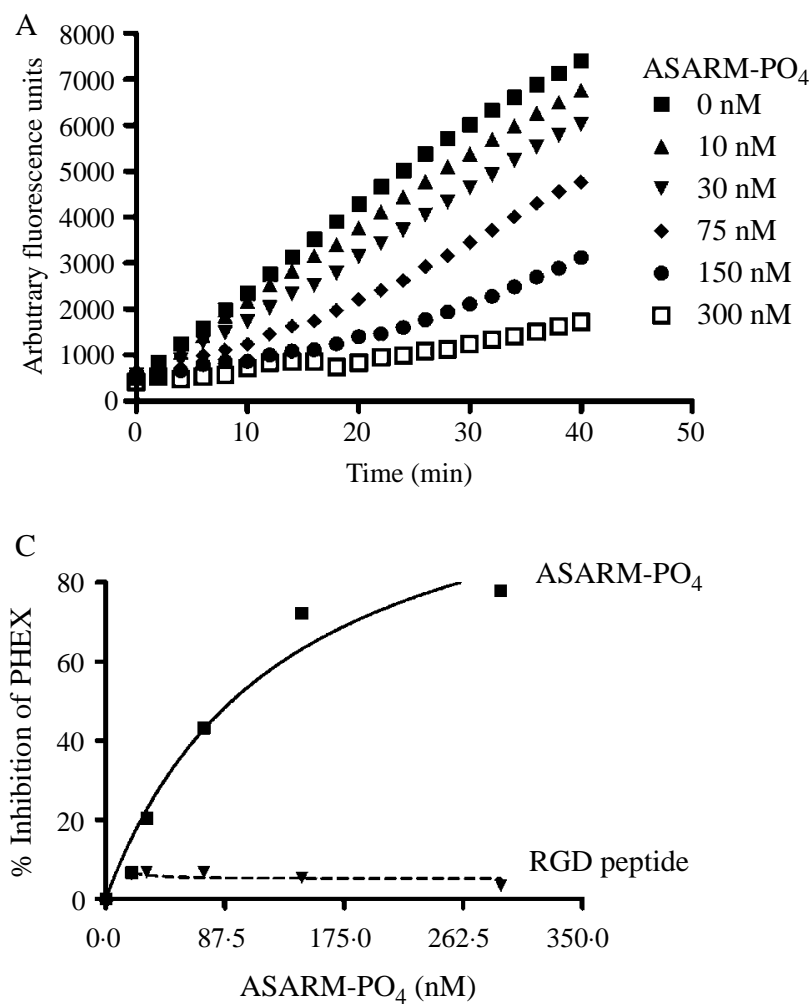

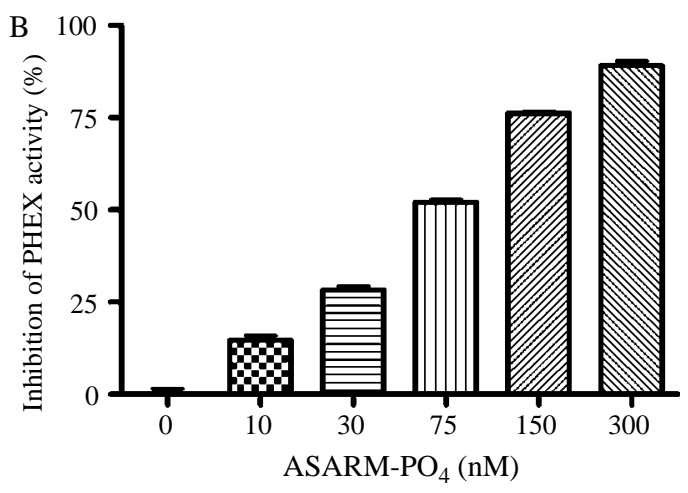

$\mathrm{D}$

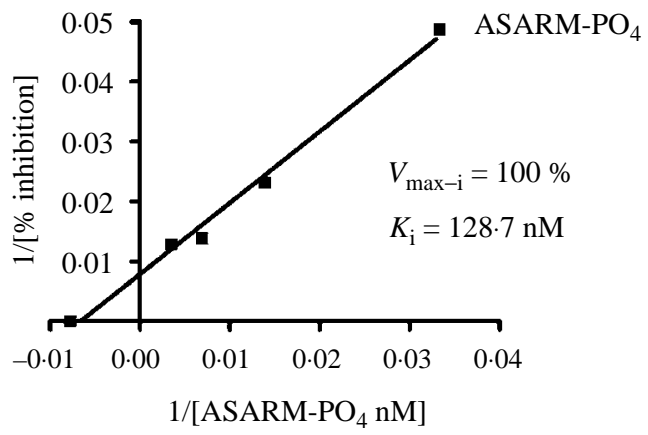

Figure 2 Kinetics of inhibition of PHEX enzymatic activity by peptides derived from MEPE. (A) Time-course of phosphorylated ASARM peptide (ASARM- $\mathrm{PO}_{4}$ ) inhibition of PHEX enzyme activity. Graph shows raw fluorescence data measurements of PHEX fluorogenic enzymatic assay in the absence and in the presence of different concentrations of ASARM-PO 4 peptide. The fluorescence strength in the reactions increased in linear relationship from 0 to $40 \mathrm{~min}$. There was a dose-dependent inhibition of PHEX by ASARM-PO 4 (10-300 nM). (B) Dose-dependent effects of ASARM-PO ${ }_{4}$ inhibition of PHEX. Percentage inhibition reflects the inhibition with peptide compared with the enzyme activity without peptide from $n=3$. (C and D) Kinetics of inhibition of PHEX enzymatic activity inhibition by ASARM-PO 4 peptide. Michaelis-Menton (C) and corresponding Lineweaver-Burke plot (D). RGD and non-phosphorylated ASARM

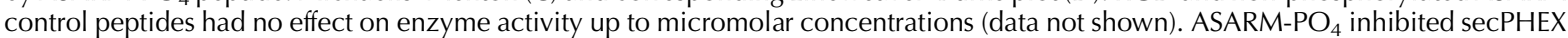
activity in a dose-dependent and saturable manner with a maximum inhibition $\left(V_{\max -\mathrm{i}}\right)$ of $100 \%$ and a $K_{\mathrm{i}}$ of $128 \cdot 7 \mathrm{nM}$. Each experimental dose point is statistically significant by one-way ANOVA analysis at $P$ value $<0 \cdot 05$.

In addition, ASARM- $\mathrm{PO}_{4}$ peptide increased eGFP expression that was limited to osteocytes embedded in mineralization nodule (Fig. 3B). However, the level of FGF23 expression was less than observed in Hyp controls.

The addition of the ASARM- $\mathrm{PO}_{4}$ peptide also inhibited mineralization, as evidenced by alizarin red-stained nodules in ASARM- $\mathrm{PO}_{4}$ peptide-treated BMSCs compared with untreated controls (Fig. 4A). In contrast to the attenuated reduction in FGF23, ASARM-PO 4 inhibited mineralization to a similar degree as Hyp-derived BMSCs (Fig. 4B).

\section{Discussion}

Our findings provide new insights into how PHEX, MEPE, and FGF23 potentially act in concert to regulate phosphate homeostasis and matrix mineralization. First, the ability of MEPE and the MEPE-derived ASARM-PO 4 peptide to inhibit PHEX activity in vitro (Fig. 2), and stimulate FGF23 production by BMSC (Fig. 3), suggests that MEPE is an important regulator of PHEX function. In previous studies, we have shown that PHEX inhibits MEPE degradation in vitro (Guo et al. 2002) and that MEPE and its ASARM$\mathrm{PO}_{4}$ peptide binds to the PHEX protein catalytic site (Rowe et al. 2005). The present findings support the possibility that the observed phosphaturic effects of MEPE in vivo (Rowe et al. 2004) are mediated, at least in part, through MEPE binding to and inactivation of PHEX. Both the ASARM$\mathrm{PO}_{4}$ peptide and MEPE maximally inhibit PHEX activity at nanomolar concentrations, in a dose-dependent and saturable manner (maximal inhibition 100\% for ASARM-PO ${ }_{4}$ and $25 \%$ for MEPE). Recent detection of micromolar levels of circulating MEPE (Bresler et al. 2004, Jain et al. 2004) suggests that the inhibition of PHEX represents a physiological concentration of MEPE. The observation that the phosphorylated ASARM peptide is more potent than full-length MEPE also raises the possibility that proteolytic processing and phosphorylation are important means to 
A

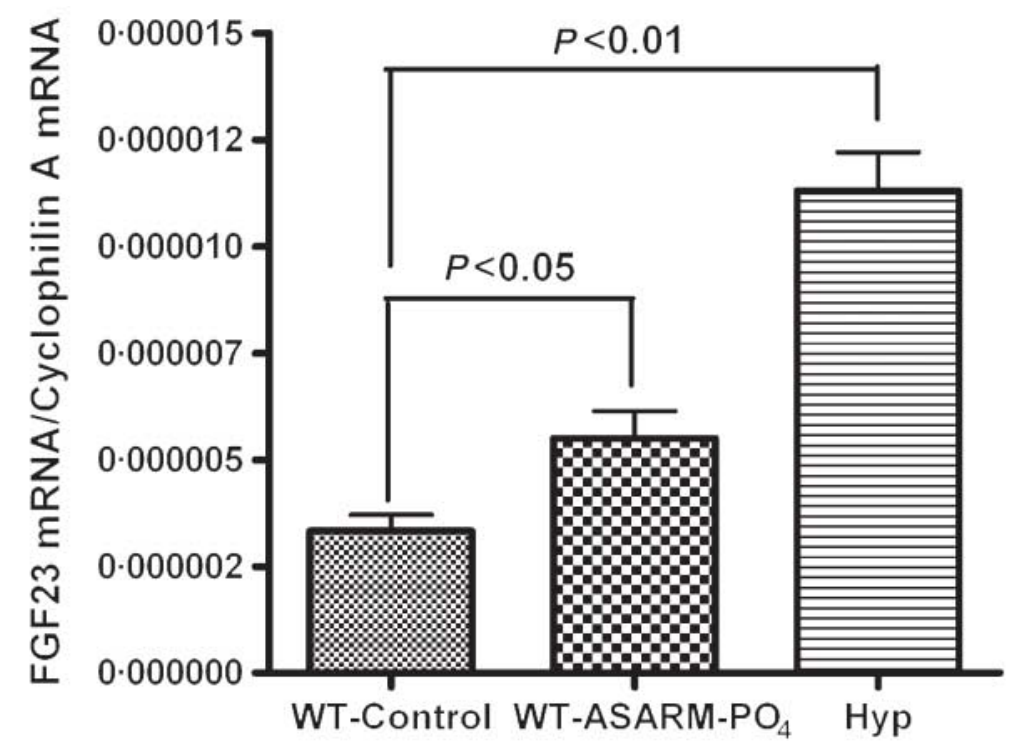

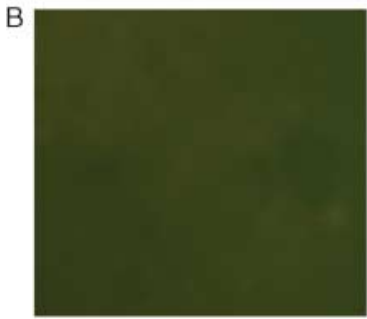

WT-control

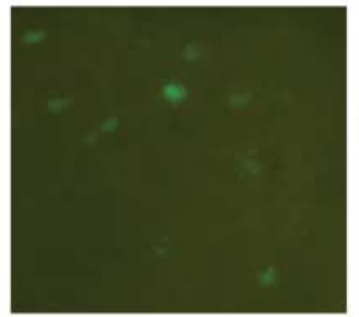

WT-ASARM-PO

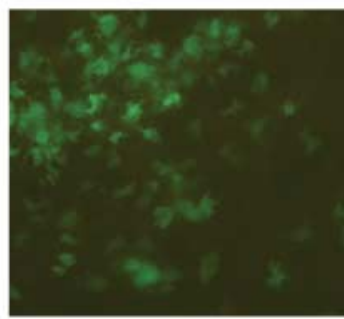

Hyp

Figure 3 Effects of ASARM-PO 4 on FGF23 expression in BMSC cultures. (A) FGF23 message levels measured by quantitative real-time RT-PCR in BMSC cultures from wild-type (controls), wild-type treated with ASARM-PO , and Hyp (positive controls). FGF23 mRNA levels are expressed relative to the levels of the cyclophilin A mRNA. Values represent the mean \pm s.E.M. $(n=3)$. ASARM- $\mathrm{PO}_{4}$ significantly stimulated FGF23 mRNA expression. (B) Assessment of ASARM-PO ${ }_{4}$ effects on eGFP expression driven by endogenous FGF23 promoter in BMSC cultures. The mineralization nodules in BMSC cultured in differentiation medium for 14 days were viewed under fluorescent microscope $(\times 200)$. WT control cultures showed no eGFP (left panel), whereas WT BMSCs cultured with the ASARM-PO ${ }_{4}$ peptide at $10 \mu \mathrm{M}$ concentration for 14 days showed demonstrable eGFP-positive cells embedded in the

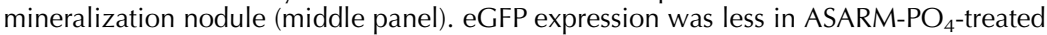
cultures compared with the abundant eGFP-positive cells in the mineralization nodules in Hyp-derived BMSCs (Hyp), which were used as positive controls (right panel).

regulate the biological functions of MEPE (Rowe et al. 2004, 2005). Recently, the importance of proteolytic processing in Hyp has been shown by the improved bone mineralization following the in vivo administration of cathepsin inhibitors (Rowe et al. 2006).

Secondly, we confirm the ability of the ASARM-PO peptide to regulate mineralization. We found that the addition of ASARM-PO ${ }_{4}$ peptide, in physiological concentrations, inhibited mineralization of BMSC cultures (Fig. 4). The MEPEderived ASARM-PO ${ }_{4}$ peptide has also been shown to inhibit mineralization in vivo in mice and in vitro using a murine osteoblast cell-line (2T3 cells; Rowe et al. 2004, 2005). Our prior observations also implicate MEPE in the mineralization defect observed in Hyp mice. In this regard, mineralization of BMSC cultures is improved in MEPE-deficient Hyp mice ex vivo, in spite of persistent hypophosphatemia in the combined MEPE-deficient/Hyp mice (Liu et al. 2005a,b). The increased MEPE in Hyp mouse bone could account for the 'intrinsic' mineralization abnormality in this disorder. Thus, MEPE and/ or its degradation products may directly interfere with the process of mineralization of the extracellular matrix independent of hypophosphatemia. The exact mechanism whereby the mineralization process is disrupted by the ASARM peptide, and the role of PHEX/MEPE interactions in the process, is not clear, but likely involves altering the ratio of inhibitors and/or inducer of mineralization (Murshed et al. 2005). 


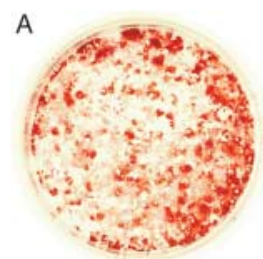

WT-control

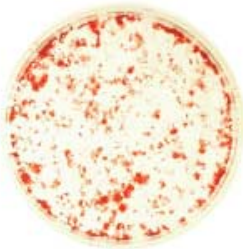

WT-ASARM-PO

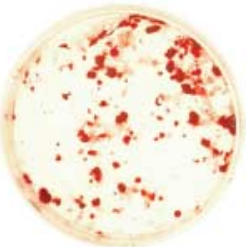

Hyp
B

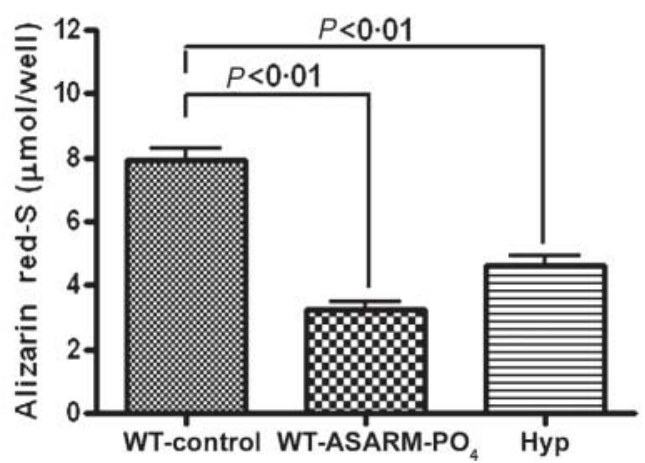

Figure 4 Effect of ASARM-PO 4 on BMSC mineralization. Alizarin Red-S staining of mineralized extracellular matrix in BMSC cultures (A) and quantification of mineralization (B). The addition of the ASARM-PO ${ }_{4}(10 \mu \mathrm{M})$ to wild-type BMSCs for 14 days resulted in less mineralization nodules similar to BMSCs derived from HyP mice. Numeric values represent the mean \pm s.E.M. of Alizarin red-S from a $35 \mathrm{~mm}$ plate $(n=3)$.

Although we demonstrate that MEPE and its ASARM peptide inhibit PHEX activity in vitro and is associated with increased FGF23 message expression in culture, our studies have not established that the reported phosphaturic actions of MEPE (Rowe et al. 2004) are mediated through increased FGF23 or inhibition of PHEX in vivo. Future studies that investigate whether FGF23-null mice are refractory to MEPE-induced phosphaturia would be necessary to establish the role of FGF23 in mediating MEPE actions. MEPE inhibition of PHEX, if physiologically relevant, places MEPE upstream of PHEX. This might explain not only the phosphaturic effect of recombinant MEPE, since excess MEPE would mimic the effects of inactivating PHEX mutations in Hyp, but potentially also explain why superimposed MEPE deficiency failed to rescue the Hyp phenotype (Liu et al. 2005b). It is also possible that MEPE has effects that are independent of both FGF23 and PHEX both in bone and other sites.

Regardless, phosphate is an important determinant of mineralization of extracellular matrix in bone and soft tissues (Murshed et al. 2005) and a bone-kidney axis involving PHEX and FGF23 may regulate renal phosphate handling to meet the demand of phosphate for mineralization of bone (Quarles 2003, Liu et al. 2006a). Our findings of the dual regulation of PHEX and mineralization by MEPE degradation products suggest that MEPE may also be involved in this putative bone-kidney axis as an intermediate molecule in bone matrix that directly regulates the mineralization process in bone and indirectly coordinates renal phosphate handling through interactions with PHEX and regulation of FGF23 production. Proof of this hypothesis will require additional studies that establish the respective roles of PHEX and FGF23 in mediating the effects of MEPE in vivo.

\section{Acknowledgements}

The authors would like to thank Dr Philippe Crine from Enobia Inc. for providing the PHEX enzymatic assay. The authors declare that there is no conflict of interest that would prejudice the impartiality of this scientific work.

\section{Funding}

This work was supported by NIH Grant Number P20 RR17708 from the National Center for Research Resources and NIH Grant Number RO1-AR45955 from the National Institute of Arthritis and Musculoskeletal and Skin Diseases to S L and D Q respectively. Also, NIH grants RO-1 AR5159801(NIAMS) and 1RO3 DE0159001 (NIDCR) to P S N R.

\section{References}

ADHR Consortium 2000 Autosomal dominant hypophosphataemic rickets is associated with mutations in FGF23. Nature Genetics 26 345-348.

Argiro L, Desbarats M, Glorieux FH \& Ecarot B 2001 Mepe, the gene encoding a tumor-secreted protein in oncogenic hypophosphatemic osteomalacia, is expressed in bone. Genomics 74 342-351.

Bai XY, Miao D, Goltzman D \& Karaplis AC 2003 The autosomal dominant hypophosphatemic rickets R176Q mutation in fibroblast growth factor 23 resists proteolytic cleavage and enhances in vivo biological potency. Journal of Biological Chemistry 278 9843-9849.

Bai X, Miao D, Li J, Goltzman D \& Karaplis AC 2004 Transgenic mice overexpressing human fibroblast growth factor 23 (R176Q) delineate a putative role for parathyroid hormone in renal phosphate wasting disorders. Endocrinology 145 5269-5279.

Benet-Pages A, Lorenz-Depiereux B, Zischka H, White KE, Econs MJ \& Strom TM 2004 FGF23 is processed by proprotein convertases but not by PHEX. Bone 35 455-462.

Bresler D, Bruder J, Mohnike K, Fraser WD \& Rowe PS 2004 Serum MEPEASARM-peptides are elevated in X-linked rickets (HYP): implications for phosphaturia and rickets. Journal of Endocrinology 183 R1-R9.

Campos M, Couture C, Hirata IY, Juliano MA, Loisel TP, Crine P, Juliano L, Boileau G \& Carmona AK 2003 Human recombinant endopeptidase PHEX has a strict $\mathrm{S}^{\prime}$ specificity for acidic residues and cleaves peptides derived from fibroblast growth factor-23 and matrix extracellular phosphoglycoprotein. Biochemical Journal 373 271-279.

Econs MJ \& Drezner MK 1994 Tumor-induced osteomalacia-unveiling a new hormone. New England Journal of Medicine 330 1679-1681.

Fisher LW \& Fedarko NS 2003 Six genes expressed in bones and teeth encode the current members of the SIBLING family of proteins. Connective Tissue Research 44(Suppl 1) 33-40.

Gowen LC, Petersen DN, Mansolf AL, Qi H, Stock JL, Tkalcevic GT, Simmons HA, Crawford DT, Chidsey-Frink KL, Ke HZ et al. 2003 Targeted disruption of the osteoblast/osteocyte factor 45 gene (OF45) results in increased bone formation and bone mass. Journal of Biological Chemistry 278 1998-2007. 
Guo R, Rowe PS, Liu S, Simpson LG, Xiao ZS \& Darryl Quarles LD 2002 Inhibition of MEPE cleavage by Phex. Biochemical and Biophysical Research Communications 297 38-45.

Jain A, Fedarko NS, Collins MT, Gelman R, Ankrom MA, Tayback M \& Fisher LW 2004 Serum levels of matrix extracellular phosphoglycoprotein (MEPE) in normal humans correlate with serum phosphorus, parathyroid hormone and bone mineral density. Journal of Clinical Endocrinology and Metabolism 89 4158-4161.

Larsson T, Marsell R, Schipani E, Ohlsson C, Ljunggren O, Tenenhouse HS, Juppner H \& Jonsson KB 2004 Transgenic mice expressing fibroblast growth factor 23 under the control of the alpha1(I) collagen promoter exhibit growth retardation, osteomalacia, and disturbed phosphate homeostasis. Endocrinology 145 3087-3094.

Larsson T, Davis SI, Garringer HJ, Mooney SD, Draman MS, Cullen MJ \& White KE 2005 Fibroblast growth factor-23 mutants causing familial tumoral calcinosis are differentially processed. Endocrinology 146 3883-3891.

Liu S, Guo R, Simpson LG, Xiao ZS, Burnham CE \& Quarles LD 2003 Regulation of fibroblastic growth factor 23 expression but not degradation by PHEX. Journal of Biological Chemistry 278 37419-37426.

Liu H, Li W, Shi S, Habelitz S, Gao C \& Denbesten P 2005a MEPE is downregulated as dental pulp stem cells differentiate. Archives of Oral Biology 50 923-928.

Liu S, Brown TA, Zhou J, Xiao ZS, Awad H, Guilak F \& Quarles LD $2005 b$ Role of matrix extracellular phosphoglycoprotein in the pathogenesis of X-linked hypophosphatemia. Journal of the American Society of Nephrology $\mathbf{1 6}$ $1645-1653$.

Liu S, Tang W, Zhou J, Stubbs JR, Luo Q, Pi M \& Quarles LD $2006 a$ Fibroblast growth factor 23 is a counter-regulatory phosphaturic hormone for vitamin D. Journal of the American Society of Nephrology 17 1305-1315.

Liu S, Zhou J, Tang W, Jiang X, Rowe DW \& Quarles LD $2006 b$ Pathogenic role of FGF23 in Hyp mice. American Journal of Physiology. Endocrinology and Metabolism 291 E38-E49.

Murshed M, Harmey D, Millan JL, McKee MD \& Karsenty G 2005 Unique coexpression in osteoblasts of broadly expressed genes accounts for the spatial restriction of ECM mineralization to bone. Genes and Development 19 1093-1104.

Petersen DN, Tkalcevic GT, Mansolf AL, Rivera-Gonzalez R \& Brown TA 2000 Identification of osteoblast/osteocyte factor 45 (OF45), a bonespecific cDNA encoding an RGD-containing protein that is highly expressed in osteoblasts and osteocytes. Journal of Biological Chemistry 275 36172-36180.

Quarles LD 2003 Evidence for a bone-kidney axis regulating phosphate homeostasis. Journal of Clinical Investigation 112 642-646.

Rowe PS, Oudet CL, Francis F, Sinding C, Pannetier S, Econs MJ, Strom TM, Meitinger T, Garabedian M, David A et al. 1997 Distribution of mutations in the PEX gene in families with X-linked hypophosphataemic rickets (HYP). Human Molecular Genetics 6 539-549.
Rowe PS, de Zoysa PA, Dong R, Wang HR, White KE, Econs MJ \& Oudet CL 2000 MEPE, a new gene expressed in bone marrow and tumors causing osteomalacia. Genomics 67 54-68.

Rowe PS, Kumagai Y, Gutierrez G, Garrett IR, Blacher R, Rosen D, Cundy J, Navvab S, Chen D, Drezner MK et al. 2004 MEPE has the properties of an osteoblastic phosphatonin and minhibin. Bone 34 303-319.

Rowe PS, Garrett IR, Schwarz PM, Carnes DL, Lafer EM, Mundy GR \& Gutierrez GE 2005 Surface plasmon resonance (SPR) confirms that MEPE binds to PHEX via the MEPE-ASARM motif: a model for impaired mineralization in X-linked rickets (HYP). Bone 36 33-46.

Rowe PS, Matsumoto N, Jo OD, Shih RN, O'Connor J, Roudier MP, Bain S, Liu S, Harrison J \& Yanagawa N 2006 Correction of the mineralization defect in hyp mice treated with protease inhibitors CA074 and pepstatin. Bone 39 773-786.

Shimada T, Mizutani S, Muto T, Yoneya T, Hino R, Takeda S, Takeuchi Y, Fujita T, Fukumoto S \& Yamashita T 2001 Cloning and characterization of FGF23 as a causative factor of tumor-induced osteomalacia. PNAS 98 6500-6505.

Shimada T, Kakitani M, Yamazaki Y, Hasegawa H, Takeuchi Y, Fujita T, Fukumoto S, Tomizuka K \& Yamashita T 2004a Targeted ablation of FGF23 demonstrates an essential physiological role of FGF23 in phosphate and vitamin D metabolism. Journal of Clinical Investigation 113 561-568.

Shimada T, Urakawa I, Yamazaki Y, Hasegawa H, Hino R, Yoneya T, Takeuchi Y, Fujita T, Fukumoto S \& Yamashita T 2004b FGF-23 transgenic mice demonstrate hypophosphatemic rickets with reduced expression of sodium phosphate cotransporter type IIa. Biochemical and Biophysical Research Communications 314 409-414.

The HYP Consortium 1995 A gene (PEX) with homologies to endopeptidases is mutated in patients with X-linked hypophosphatemic rickets. Nature Genetics 11 130-136.

Weber TJ, Liu S, Indridason OS \& Quarles LD 2003 Serum FGF23 levels in normal and disordered phosphorus homeostasis. Journal of Bone and Mineral Research 18 1227-1234.

White KE, Carn G, Lorenz-Depiereux B, Benet-Pages A, Strom TM \& Econs MJ 2001 Autosomal-dominant hypophosphatemic rickets (ADHR) mutations stabilize FGF-23. Kidney International 60 2079-2086.

Yamazaki Y, Okazaki R, Shibata M, Hasegawa Y, Satoh K, Tajima T, Takeuchi Y, Fujita T, Nakahara K, Yamashita T et al. 2002 Increased circulatory level of biologically active full-length FGF-23 in patients with hypophosphatemic rickets/osteomalacia. Journal of Clinical Endocrinology and Metabolism 87 4957-4960.

\section{Received in final form 4 October 2006 Accepted 13 October 2006 Made available online as an Accepted Preprint 17 October 2006}

